\title{
ARTICLE OPEN Arabinoxylans, inulin and Lactobacillus reuteri 1063 repress the adherent-invasive Escherichia coli from mucus in a mucosa-comprising gut model
}

\author{
Pieter Van den Abbeele ${ }^{1,3}$, Massimo Marzorati ${ }^{1,3}$, Melanie Derde ${ }^{1}$, Rosemarie De Weirdt ${ }^{1}$, Vermeiren Joan ${ }^{1}$, Sam Possemiers ${ }^{1,2}$ \\ and Tom Van de Wiele ${ }^{1}$
}

The microbiota that colonises the intestinal mucus may particularly affect human health given its proximity to the epithelium. For instance, the presence of the adherent-invasive Escherichia coli (AIEC) in this mucosal microbiota has been correlated with Crohn's disease. Using short-term screening assays and a novel long-term dynamic gut model, which comprises a simulated mucosal environment (M-SHIME), we investigated how (potential) pro- and prebiotics may repress colonisation of AIEC from mucus. Despite that during the short-term screening assays, some of the investigated Lactobacillus strains adhered strongly to mucins, none of them competed with AIEC for mucin-adhesion. In contrast, AIEC survival and growth during co-culture batch incubations was decreased by Lactobacillus rhamnosus GG and L. reuteri 1063, which correlated with (undissociated) lactic acid and reuterin levels. Regarding the prebiotics, long-chain arabinoxylans (LC-AX) lowered the initial mucin-adhesion of AIEC, while both inulin (IN) and galacto-oligosaccharides (GOS) limited AIEC survival and growth during batch incubations. L. reuteri 1063, LC-AX and IN were thus retained for a long-term study with the M-SHIME. All treatments repressed AIEC from mucus without affecting AIEC numbers in the luminal content. As a possible explanation, L. reuteri 1063 treatment increased lactobacilli levels in mucus, while LC-AX and IN additionally increased mucosal bifidobacteria levels, thus leading to antimicrobial effects against AIEC in mucus. Overall, this study shows that pro- and prebiotics can beneficially modulate the in vitro mucosal microbiota, thus limiting occurrence of opportunistic pathogens among those mucosal microbes which may directly interact with the host given their proximity to the epithelium.

npj Biofilms and Microbiomes (2016) 2, 16016; doi:10.1038/npjbiofilms.2016.16; published online 27 July 2016

\section{INTRODUCTION}

Along the intestinal tract, the host epithelium is covered by a protective mucus layer that contains specific microbes ${ }^{1}$ and several factors contribute to a distinct microbiota in the luminal content versus the mucus layer. ${ }^{2}$ First, the host selects microbes that colonise mucus by producing defence molecules such as antimicrobial peptides (AMPs) and $\operatorname{lgA}{ }^{3}$ Selection also occurs through oxygen diffusion from the blood towards the gut, resulting in an oxygen gradient along the mucus. The unique mucosal microbiota composition is further determined by bacterial factors such as mucus adhesion ${ }^{4}$ and mucin degradation..$^{5}$ The resulting mucosal microbiota as such possesses a colonisation resistance against opportunistic pathogens. This includes local excretion of antimicrobial compounds, ${ }^{6}$ stimulation of the host immune system, ${ }^{7}$ production of metabolic compounds that lower the $\mathrm{pH}^{8}$ and competition with pathogens for nutrients ${ }^{9}$ and adhesion sites. ${ }^{10}$

A disruption of the mucosal microbial community may have adverse implications for human health. Besides an altered interaction with the immune system, a disrupted mucosal microbiota may result in increased colonisation by (potential) pathogens correlated with infectious or chronic gastrointestinal diseases. As an example, the mucosal microbiota of Crohn's disease $(C D)$ patients is characterised by the dominance of potentially harmful microbes, in particular Escherichia coli, over beneficial microbes such as Faecalibacterium prausnitzii and Roseburia sp. ${ }^{11,12}$ In fact, the adherent-invasive Escherichia coli (AIEC), a strain proposed as a causative agent of CD, can strongly adhere to and invade intestinal epithelial cells in patients by means of a protease called Vat-AIEC. ${ }^{13,14}$

While in vivo studies are restricted to end-point measurements regarding mucosal microbes, ${ }^{1}$ in vitro assays allow to study mechanisms of microbial adhesion. These assays include adhesion experiments to mucus, ${ }^{15}$ mucins, ${ }^{16}$ colonic tissue ${ }^{17}$ and cell lines. ${ }^{18}$ Because these models only provide short-term information and ignore the interaction between luminal and mucosal microbes, we modified a dynamic in vitro model for the luminal microbiota by incorporating mucin-covered microcosms (M-SHIME). The validation of this M-SHIME model followed from the more representative colonisation of Lactobacillus sp. ${ }^{19}$ Furthermore, high-resolution phylogenetic characterisation showed that the simulated mucosal microbiota was, in correspondence with in vivo studies, enriched with Firmicutes sp. belonging to Clostridium clusters IV and XIVa. ${ }^{20}$ The same study showed that the in vitro mucosal environment is necessary to avoid wash-out of relevant surface-attached microbes.

As reinforcing the mucosal microbiota may be a strategy to restore the host-microbe interaction when disturbed, the objective of this study was to evaluate the efficacy of several

\footnotetext{
${ }^{1}$ Center of Microbial Ecology and Technology (CMET), Ghent University, Ghent, Belgium and ${ }^{2}$ ProDigest, Ghent, Belgium.

Correspondence: T Van de Wiele (tom.vandewiele@ugent.be)

${ }^{3}$ These authors contributed equally to this work.

Received 31 March 2016; revised 19 May 2016; accepted 3 June 2016
} 
strategies to enhance the mucosal microbiota in repressing opportunistic pathogens from the mucosal environment. To demonstrate the proof-of-principle, we used AIEC as a model opportunistic pathogen, as AIEC is known to strongly adhere to mucus in $C D$ patients, while the ampicillin/erythromycin-resistant reference strain LF82 ${ }^{21}$ enables straightforward detection of viable AIEC cells in the background of a mixed intestinal microbiota. Using short-term screening assays, we evaluated how selected pre- and probiotics may inhibit the initial adhesion of AIEC to intestinal mucins, as well as the survival/growth of AIEC. A selection of treatments was then applied in the M-SHIME to investigate whether AIEC was repressed in a simulated mucosal environment during a long-term interaction with the resident luminal and mucosal microbiota of a single donor.

\section{RESULTS}

Short-term mucin-adhesion experiments

As inhibition of AIEC adhesion by Lactobacillus strains may depend on competition for mucin-binding sites, the intrinsic adhesion capacity of the probiotic strains was evaluated in a first experiment. This showed that the strains significantly differed in their intrinsic adhesion capacity (Figure 1a). While the adhesion capacity of Lactobacillus rhamnosus GG was strong and comparable to that of AIEC ( 20\%), Pediococcus acidilactici LB1 adhered poorly ( 3\%). Adhesion of L. mucosae LB2, L. reuteri 1063 and L. acidophilus NCFM was intermediate ( 10\%). However, none of the tested Lactobacillus strains lowered adhesion of AIEC in competition experiments, as compared with the AIEC control without probiotics (Figure 1b).

In contrast, two prebiotic compounds interfered with the initial adhesion of AIEC to mucins (Figure 1c). While fructooligosaccharides (FOS) and inulin (IN) had no influence, long-chain arabinoxylan (LC-AX) significantly inhibited adhesion of AIEC $(P=0.008)$. Finally, in presence of galacto-oligosaccharides (GOS), the adhesion of AIEC was significantly higher $(P=0.009)$.

\section{Short-term survival/growth inhibition assay}

On inoculation of AIEC in the nutritional medium, a good growth was monitored with a factor 60 increase in the control after $24 \mathrm{~h}$. All strains tested, except L. mucosae LB2, inhibited AIEC survival/growth (Table 1). AIEC numbers after 24-h incubation were significantly lower-as compared with the control-in the presence of $P$. acidilactici LB1, L. acidophilus NCFM, L. rhamnosus GG and $L$. reuteri 1063 . For these last two strains, a complete eradication of AIEC below detection limit was observed. As AIEC is able to produce both acetate and ammonium, these metabolites are potential markers for AIEC growth, even if it has to be taken into account that these metabolites can also remain high due to growth of other species. Acetate levels correlated with AIEC growth/survival, with the exception of the co-culture with $L$. reuteri 1063, in which acetate levels remained high despite the fact that AIEC was eradicated. Ammonium levels only decreased for the treatments that eradicated AIEC below detection limit, i.e., L. reuteri 1063 and L. rhamnosus GG. To provide an explanation for the observed antimicrobial effects, the $\mathrm{pH}$ decrease and concentrations of D/L-lactic acid (and its antimicrobial fraction, i.e., undissociated lactic acid), 3-HPA and 1,3-PDO (markers for reuterin) were determined. It followed that a decrease in AIEC numbers corresponded with lower $\mathrm{pH}$ and increased levels of undissociated lactic acid. The strong antimicrobial effect of L. reuteri 1063 was plausibly due to the combined action of undissociated L-lactic acid and reuterin (approximate increase of 3-HPA and 1,3-PDO), while in the case of L. rhamnosus GG it was related to high levels of undissociated L-lactic acid.

The indirect antimicrobial effect of oligo- and polysaccharides has been evaluated in relation to the production of metabolites
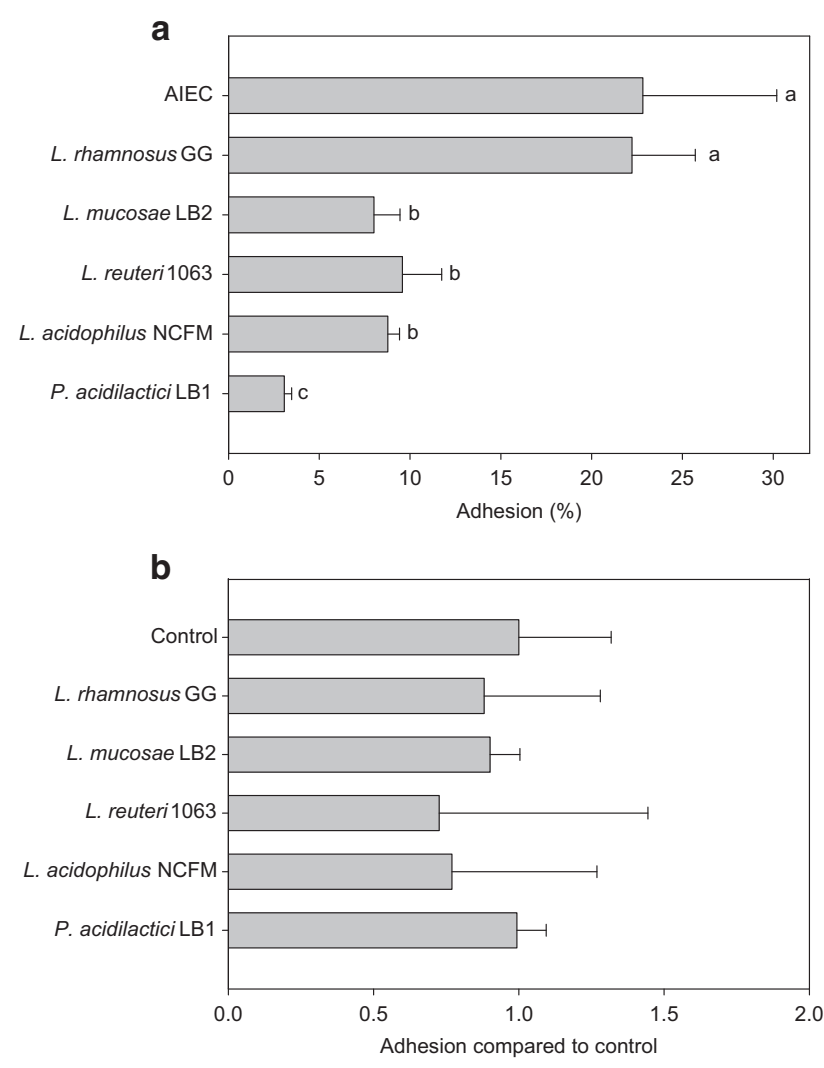

C

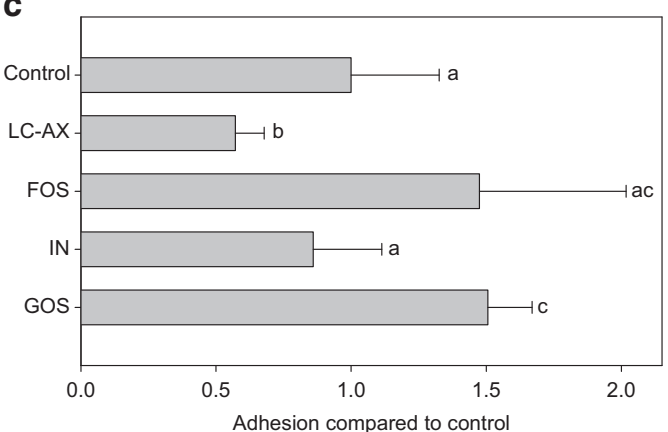

Figure 1. (a) Mean intrinsic mucin-adhesion capacity of AIEC ( \pm s.d.) $(n=16)$ and several Lactobacillus strains $(n=4)$, expressed as a ratio of the amounts of adhered bacteria compared with initially added bacteria $\left(10^{8}\right.$ cells per $\left.\mathrm{ml}\right)$. (b) Adhesion of AIEC in the presence of several Lactobacillus strains, as a ratio compared with the control where only AIEC was added $(n=4)$. (c) Adhesion of AIEC in the presence of several prebiotic compounds (LC-AX; $n=7$, FOS; $n=4$, IN; $n=4$ and GOS; $n=4$ ), as a ratio compared with the control where only AIEC was added $(n=11)$. Values indicated with a different superscript are significantly different $(P \leqslant 0.05 ; \mathrm{a}, \mathrm{b}$ or $\mathrm{c})$.

resulting from microbial catabolism. In this respect, the nutritional medium was inoculated with AIEC and also with $5 \times 10^{6}$ c.f.u. per $\mathrm{ml}$ of an ascending colon suspension from a SHIME system (control B). It was found that this mixed microbiota already exerted a vast inhibitory effect on AIEC growth (approximately only factor 0.27 increase after $24 \mathrm{~h}$ in control B in contrast to control A without mixed microbiota, which showed a $57.2 \pm 19.7$-fold increase) (Table 2). Both GOS and IN further enhanced the inhibitory effect of this mixed microbiota against AIEC, while LC-AX did not affect AIEC. For GOS, the decrease in AIEC levels coincided with a lower $\mathrm{pH}$ and more undissociated lactic acid, while for IN higher levels of 1,3-PDO and thus potentially more reuterin were observed as opposed to the other 
Table 1. The increase in AIEC numbers after 24-h incubation with different Lactobacillus sp., as a ratio compared with the initial AIEC count $\left(5 \times 10^{6}\right.$ c.f.u. per $\left.\mathrm{ml}\right)(n=3)$

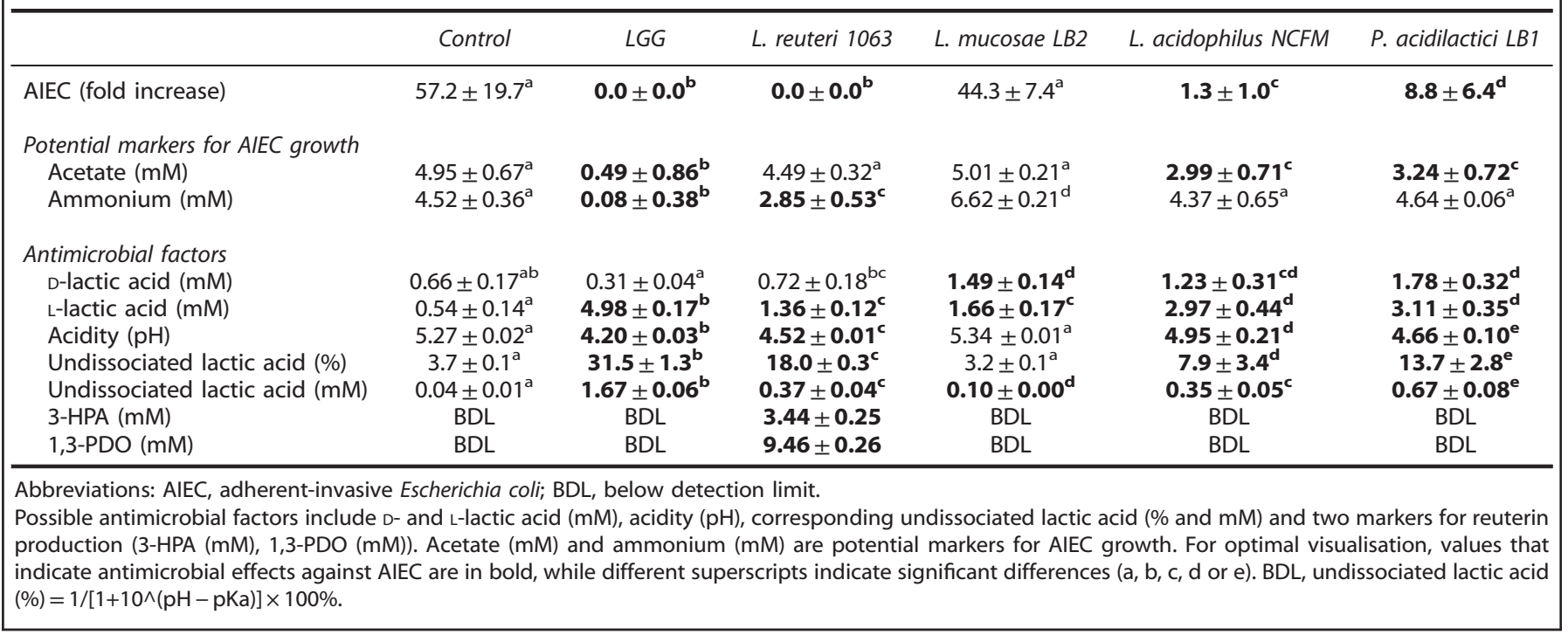

Table 2. The increase in AIEC numbers after 24-h incubation in nutritional medium without (control A) or with addition of an equal amount of a mixed SHIME-derived microbiota (control B) treated with prebiotic compounds (LC-AX, FOS, IN and GOS), expressed as a ratio compared with the initial AIEC count $\left(5 \times 10^{6}\right.$ c.f.u. per $\left.\mathrm{ml}\right)(n=5)$

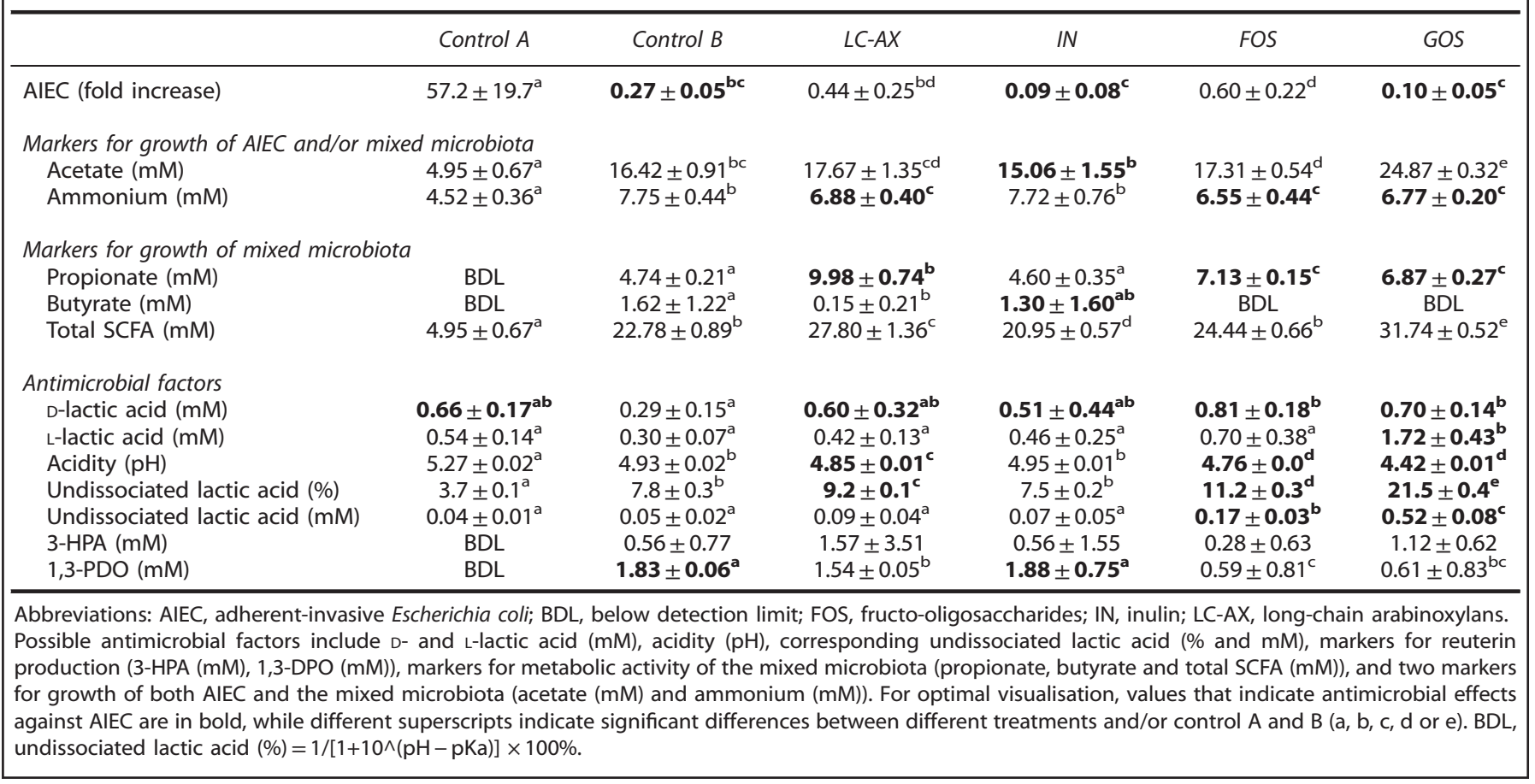

prebiotics. Despite elevated levels of undissociated lactic acid, FOS inhibited the growth of AIEC less than what is seen for control B. Given the metabolic activity of the mixed microbiota, acetate and ammonium were no optimal markers for AIEC growth. Together with propionate, butyrate and the total SCFA levels, they rather indicated the growth of the mixed intestinal microbiota.

\section{Long-term M-SHIME study}

Based on initial screenings, three treatments were applied in a follow-up M-SHIME experiment (i.e., L. reuteri 1063, LC-AX and IN) with the aim of understanding whether AIEC is repressed from a simulated mucosal environment during a long-term interaction with the resident luminal and mucosal microbiota.

The AIEC numbers (c.f.u. per $\mathrm{ml}$ ) in the luminal environment were similar between the different ascending colon units of the M-SHIME, but tended to be lower in that of the L-SHIME, which lacks a mucosal environment (Figure 2a). In contrast, AIEC counts within the mucosal environment were at least 1-log unit lower as compared with the control as a result of the treatments in the M-SHIME (Figure 2b). On inoculation of AIEC (on day 22, 23, 24 and 25), LC-AX immediately decreased AIEC in the mucus (day 24). During further treatment, supplementation of $L$. reuteri 1063, LC-AX and IN was similarly effective in lowering AIEC numbers in 


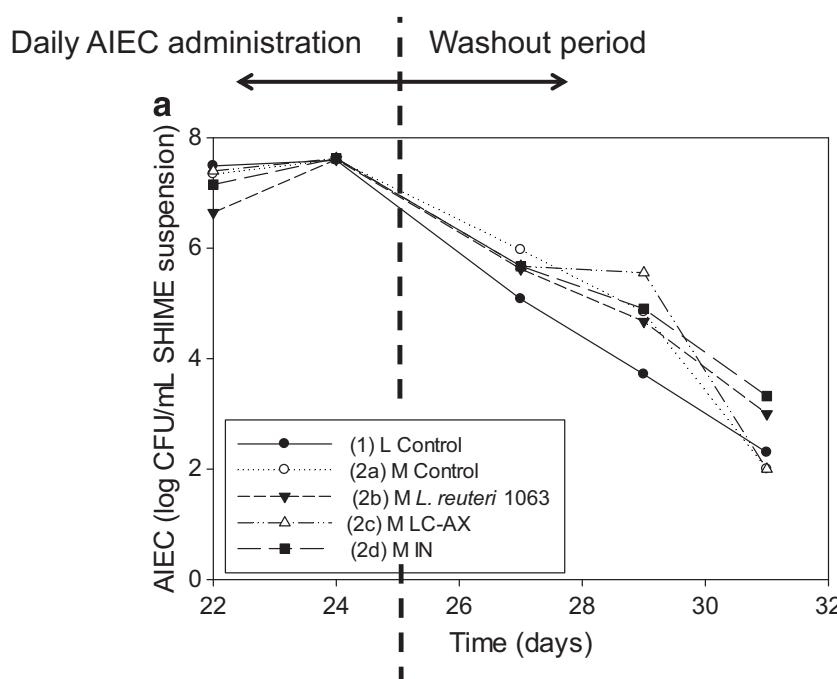

B. adolescentis in mucus. IN led also to a delayed stimulation (i.e., day 29) of B. adolescentis in mucus and lumen (Supplementary Figure 1B). In addition, L. reuteri 1063 elevated lactic acid and reuterin ( 1,3-PDO) levels, while LC-AX and IN only increased reuterin production ( 1,3-PDO) (Table 3$)$.

\section{DISCUSSION}

In this study, we provide an in vitro proof-of-principle that pre- and probiotic strategies can beneficially modulate the mucosal microbiota and decrease the mucosal colonisation of an opportunistic pathogen (i.e., Escherichia coli AIEC). Because the mucosal microbes may directly interact with the host, lowering the abundance of opportunistic pathogens in the mucosal compartment may be more relevant than lowering their abundance in the intestinal content. We used the AIEC as a model opportunistic pathogen because it is known to adhere strongly to mucus and because its reference strain LF82 can be easily detected in a mixed microbiota. ${ }^{21}$ As the ileal microbiota is difficult to simulate in vitro due to the lack of a representative inoculum and the difficulty of simulating the host factors that govern ileal composition (e.g., secretion of AMPs, high flow through), we simulated the ascending colon considering that AIEC are associated both with ileal and colonic disease phenotypes. ${ }^{14}$ After short-term screening assays, a selection of pro- and prebiotics was applied in a dynamic in vitro model (M-SHIME) that allows to study both the luminal and mucosal intestinal microbiota. ${ }^{19}$ This simulated mucosal environment avoids wash-out of specific surface-associated microbes. ${ }^{20}$ As AIEC also benefits from mucus adhesion to colonise the human intestine, this novel model allowed studying the colonisation of AIEC in a more representative manner compared with earlier models.

The initial mucin-adhesion capacity of AIEC was strong and it was comparable to that of well-known mucus colonisers such as L. rhamnosus $\mathrm{GG}^{23}$ and $L$. reuteri $1063^{4}$ (Figure 1a). The mucinadhesion of AIEC was confirmed during the long-term experiment, where AIEC was an abundant member of the mucosal microbiota, even 6 days after its last inoculation (day 31; Figure 2b). The ability of AIEC to colonise the intestinal surface has been attributed to active motility through flagella which also regulate expression of type 1 pili. $^{24}$ For AIEC, mucus colonisation is crucial to adhere to and invade host cells. ${ }^{13,25}$

L. reuteri 1063, LC-AX and IN specifically lowered AIEC numbers in the simulated mucosal environment of the M-SHIME, while they did not affect AIEC numbers in the luminal content. As a possible explanation, supplementation of IN and LC-AX (during 8 days prior to AIEC inoculation) altered the resident mucosal microbiota, especially subdominant groups (lactobacilli and bifidobacteria). Not only did LC-AX and IN increase the mucosal counts of lactobacilli and bifidobacteria, they also altered the species composition of the bifidobacteria. As shown by DGGE, initially the dominant mucosal Bifidobacterium species was $B$. bifidum, IN specifically stimulated $B$. adolescentis in mucus and LC-AX specifically stimulated both $B$. longum and $B$. adolescentis in mucus. $B$. longum has been shown to possess a stronger antimicrobial activity against Escherichia coli compared with $B$. bifidum. ${ }^{26}$ Also $B$. adolescentis was shown to be very effective in combating Escherichia coli compared with B. bifidum ${ }^{27}$ but also compared with many other Bifidobacterium and Lactobacillus species. ${ }^{28}$ While antimicrobial factors are possibly too diluted in the intestinal content to be effective, the mucosal environment may allow trapping of antimicrobial factors, thereby repressing AIEC. Especially the spatial heterogeneity introduced by the biofilm on top of the mucin layer may result in local accumulation of e.g., acids produced by lactobacilli or bifidobacteria.

Although the short-term screening assays are inevitably confounded by drawbacks such as $\mathrm{pH}$ drops, nutrient limitation and accumulation of metabolites, they allowed to demonstrate 
Table 3. The average levels and s.d. of possible antimicrobial factors on time points after AIEC administration during the long-term M-SHIME experiment (day 22-31): D- and L-lactic acid (mM), markers for reuterin production (3-HPA (mM) and 1,3-PDO (mM)) and the amount of bifidobacteria and lactobacilli $(\log 10$ c.f.u. per $\mathrm{ml}$ ), both in lumen and mucus

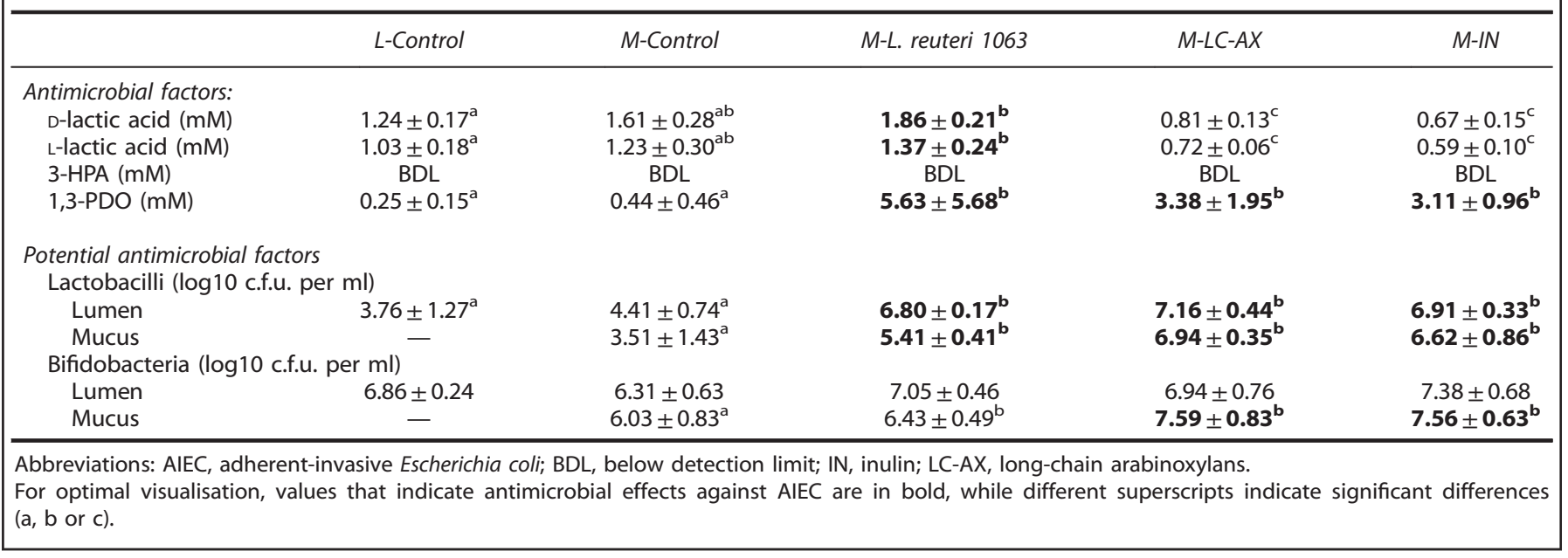

that both the conversion of glycerol to reuterin and the undissociated lactic acid production are processes that exert distinct antimicrobial effects on AIEC (Tables 1 and 2). As shown for LGG and GOS, the antimicrobial effect of lactic acid is dependent on a concomitant $\mathrm{pH}$ decrease as this enhances the portion of undissociated lactic acid. Only undissociated lactic acid can pass through the cell wall after which it exerts an antimicrobial effect by releasing protons in the cytoplasm of AIEC. While the $\mathrm{pH}$ was controlled in the intestinal lumen of the M-SHIME ( $\mathrm{pH}=5.6-5.9)$, the mucosal $\mathrm{pH}$ may have decreased below 5.6 resulting in higher mucosal levels of undissociated lactic acid (>1.79\%). Further, for L. reuteri 1063 and the mixed intestinal microbiota, the presence of the glycerol conversion products, 3-HPA and 1,3-PDO, indicated the production of the potent, broad-spectrum antimicrobial reuterin capable of inhibiting growth of many other microbial species, including Escherichia spp. ${ }^{29-32}$ As glycerol levels are higher in CD patients ${ }^{33}$ and in the upper digestive tract, the conversion of glycerol to reuterin may be particularly relevant for AIEC eradication.

Another mechanism that may result in lower mucosal AIEC counts is an adhesion inhibition of AIEC to intestinal mucins. However, among all (potential) pro- and prebiotics tested (even the strongly adherent probiotics), LC-AX was the only treatment that lowered the initial AIEC adhesion (Figure $1 b, c$ ). Also during the long-term M-SHIME study, only LC-AX limited the initial colonisation of the mucosal environment by AIEC (Figure 2b). These results are concordant with earlier studies showing that this specific LC-AX can lower the initial mucin-adhesion of a wide variety of bacterial groups including coliforms. ${ }^{16}$ The latter study demonstrated that the underlying reason may be that LC-AX increase the viscosity. Further, physical adhesion inhibition may occur by binding of microbes to fibres instead of surface receptors. $^{34}$ LC-AX-type polysaccharides may thus be interesting compounds to modulate the initial bacterial adhesion to mucins.

Despite the fact that the mucosal environment was partly (50\%) renewed every 2 days, specific species cross-contaminated between old and new mucin-microcosms, resulting in a distinct microbiota in the luminal and mucosal environment (Supplementary Figure 1). In previous studies with the M-SHIME, where the mucosal environment was not renewed, even stronger differences between the mucosal and luminal microbiota were observed on day $1(\sim 60 \% \text { similarity })^{19}$ or day 3 after start-up (only $\sim 15 \%$ similarity). ${ }^{20}$

In conclusion, we showed that the M-SHIME technology-using mucin-covered microcosms-provided a detailed insight in the long-term in vitro microbial colonisation of AIEC, an opportunistic pathogen and abundant mucosal microbe. Moreover, it allowed to evaluate the colonisation of a simulated mucus layer in the presence of a resident mucosal and luminal intestinal microbiota. It has to be considered that in this type of studies, the relevance of the data in terms of potential interindividual variability (i.e., different effect of the test products due to a different composition of the gut microbiota) may be questionable. Our aim was to present a technology platform that might be applied on other disease-causing microbes such as food-borne pathogens and to provide evidences on the potential of several pre- and probiotic strategies to repress AIEC from the mucosal compartment. Here we showed-with the microbiota from one donor-that such repression may occur via different mechanisms including the production of reuterin, undissociated lactic acid or adhesion inhibition. The evaluation of the role of the microbiota from different donors on these mechanisms can be an interesting future line of research. We also showed that the incorporation of a mucosal environment in dynamic gut models may be a powerful tool to obtain a more realistic view on processes that drive the gastrointestinal microbiome.

\section{MATERIALS AND METHODS}

Preparation of growth media and bacterial suspensions

Unless stated otherwise, chemicals were obtained from Sigma (Bornem, Belgium). Cosucra (Warcoing, Belgium) provided FOS with a purity of $96 \%$ and a degree of polymerisation (DP) between 2 and 20 (Fibrulose F97) and IN with a purity of $92 \%$ and a DP between 3 and 60 (Fibruline instant). Friesland Campina Domo (Amersfoort, the Netherlands) provided GOS with a purity of $98 \%$ and a DP between 3 and 8 (Vivinal GOS, Friesland Campina Domo). BioActor (Maastricht, the Netherlands) provided water-extractable LC-AX with a purity of $60 \%$, a degree of substitution of 0.7 and an average $\mathrm{DP} \geqslant 60$.

Ampicillin/erythromycin-resistant AIEC LF82, isolated from a chronic ileal lesion of a CD patient, was used as the AIEC reference strain..$^{21}$ Following strains were used during this study: L. rhamnosus GG (LMG 18243), L. reuteri 1063 (ATCC 53608), L. acidophilus NCFM (Danisco, Brugge, Belgium), L. mucosae LB2 ${ }^{19}$ and P. acidilactici LB1. ${ }^{19}$ Pure cultures of Lactobacillus sp. were grown overnight in MRS medium (Oxoid, Cambridge, UK), while AIEC was grown in $\mathrm{BHI}$ medium (Oxoid), both at $37^{\circ} \mathrm{C}$ under aerobic conditions.

The nutritional medium of the M-SHIME consisted of (in $\mathrm{g} / \mathrm{l}$ ) arabinogalactan (1.0), pectin (2.0), xylan (1.0), starch (3.0), glucose $(0.4)$, yeast extract (3.0), peptone (1.0), mucin (4.0) and cystein (0.5). Glycerol (1.0) was added to enable $L$. reuteri among others to produce reuterin, a bacteriocin. ${ }^{35}$ Pancreatic juice contained (in $\mathrm{g} / \mathrm{l}$ ) $\mathrm{NaHCO}_{3}$ (12.5), bile salts (6.0) (Difco, Bierbeek, Belgium) and pancreatin (0.9). 
-.... Headspace connections

_ Liquid connection

( Pump

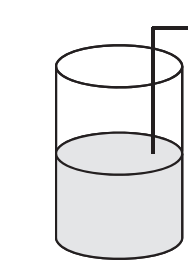

Pancreatic juice

Q

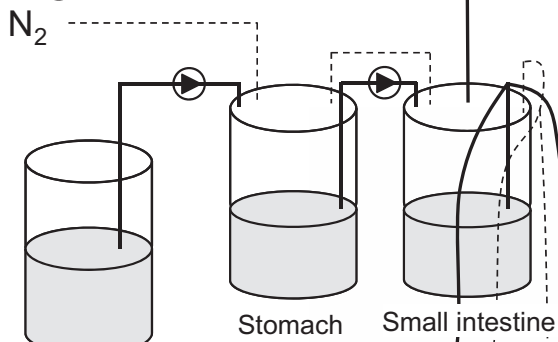

5 ascending colon vessels:
SHIME feed

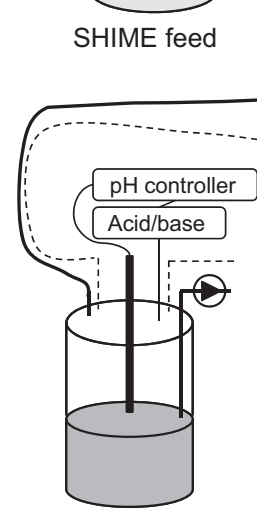

(1) Control

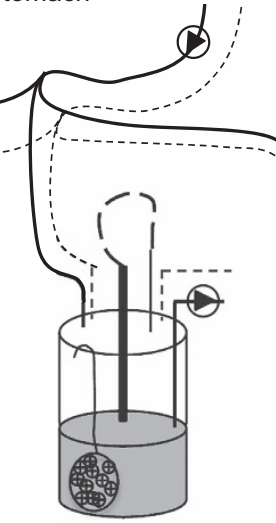

(2a) Control
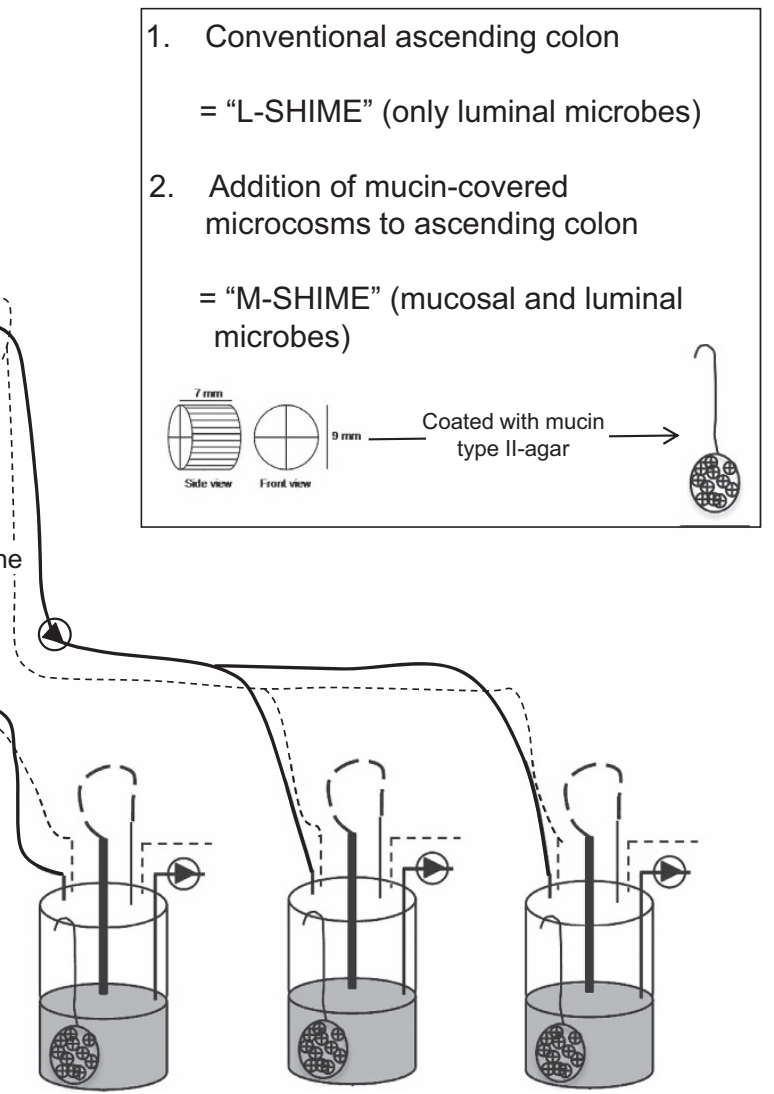

(2b) Lactobacillus reuteri

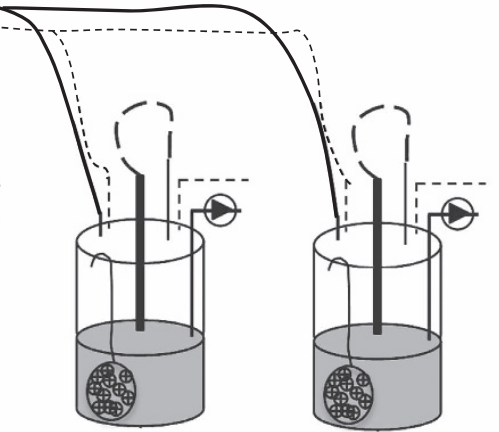

(2c) LC-AX

(2d) IN

Figure 3. The experimental design was based on the Simulator of the Human Intestinal Microbial Ecosystem (SHIME), a dynamic in vitro model of the human gastrointestinal tract. In this experiment, only the first colon compartment (ascending colon) was used and five compartments were run in parallel. The first unit consisted of a conventional setup that only contains luminal microbes (=luminal SHIME or L-SHIME (1)), whereas the other four units were modified by incorporating a mucosal compartment (= mucosal SHIME or M-SHIME (2a) (2b) (2c) (2d)), which contained 20 mucin-covered microcosms per $100 \mathrm{ml}$ suspension. The three last units were treated with L. reuteri 1063, LC-AX and IN, respectively.

\section{Short-term AIEC mucin-adhesion assay}

In a first experiment, the intrinsic adhesion capacity of AIEC and the Lactobacillus strains to mucins was determined. Moreover, inhibition of AIEC adhesion by these (potential) probiotics and prebiotics (FOS, IN, GOS and LC-AX) was evaluated. The mucin-adhesion assay was performed as described previously. ${ }^{16}$ Briefly, an overnight culture was diluted in fresh growth medium (1:10) and allowed to grow for another $3 \mathrm{~h}$. Bacterial cells were washed three times with filter-sterilised $0.1 \mathrm{M}$ phosphate-buffered saline (PBS) at pH 5.9 and diluted to a final density of $\sim 10^{8}$ cells per $\mathrm{ml}$ (using flow cytometry on live-dead staining). Immediately thereafter, $1 \mathrm{ml}$ of bacterial suspension and $1 \mathrm{ml}$ of PBS were added to 12-well plates covered with $1.2 \mathrm{ml}$ mucin agar. Mucin agar was prepared by boiling distilled $\mathrm{H}_{2} \mathrm{O}$ containing $5 \%$ gastric porcine mucin type II (Sigma) and 1\% agar. The $\mathrm{pH}$ was adjusted to 6.8 with $10 \mathrm{M} \mathrm{NaOH}(\sim 350 \mu \mathrm{l}$ per $100 \mathrm{ml})$. Bacteria were allowed to adhere to this mucin layer under anaerobic conditions, at $37^{\circ} \mathrm{C}$ and under slight agitation (30 r.p.m.). After 80 -min incubation, non-adhered bacteria were removed, each well was washed three times with PBS and the remaining adhered bacteria were detached using $0.5 \%$ Triton X-100 in PBS. The total amount of initially added and finally adhered bacteria was quantified using flow cytometry. To evaluate the inhibitory effect of a pro- or prebiotic on adhesion of AIEC, the extra $1 \mathrm{ml}$ of PBS was supplemented with $10^{8}$ probiotic cells per $\mathrm{ml}$ or $15 \mathrm{~g} / \mathrm{l}$ of the prebiotic compound. When co-cultured, the initially added and finally adhered amounts of AIEC were determined using plate counts.

\section{Short-term AIEC survival and growth inhibition assay}

In a second experiment, the inhibitory effect of the selected pro- and prebiotics on the survival/growth of AIEC in nutritional medium (initial
$\mathrm{pH}=6.0$ ) was evaluated during batch incubations. To do this, $15 \mathrm{ml}$ nutritional medium was added to penicillin bottles in which anaerobic conditions were obtained flushing with N2 during 15 cycles of 2 min each at 800 mbar over-pressure and 900 mbar under-pressure. To test the inhibitory effect of the probiotics, $5 \times 10^{6}$ cells per $\mathrm{ml}$ of both the probiotic and AIEC were added. To test the inhibitory effect of prebiotics, $10 \mathrm{~g} / \mathrm{l}$ prebiotic (control-starch) was added to SHIME nutritional medium containing $5 \times 10^{6}$ cells per $\mathrm{ml}$ of both AIEC and additionally a mixed microbiota from the ascending colon of the SHIME $(\sim 10 \mu \mathrm{l}$ colon suspension). This mixed microbiota was only added during the assays with prebiotics as prebiotic compounds rather inhibit growth of AIEC indirectly through stimulation of other microbes. Penicillin bottles were incubated at $37^{\circ} \mathrm{C}$ and 140 r.p.m. for $24 \mathrm{~h}$. At the final time point, $\mathrm{pH}$, D/L-lactic acid, reuterin, 1,3-PDO, SCFA, $\mathrm{NH}_{4}$ and counts of AIEC and the probiotic were determined (c.f.u. per $\mathrm{ml}$ ).

\section{Dynamic gut model (M-SHIME)}

Recently, we developed an in vitro model for the human intestinal tract which accounts for both luminal and mucosal microbes, i.e., the M-SHIME, based on the validated Simulator of the Human Intestinal Microbial Ecosystem (SHIME, Ghent University-ProDigest, Belgium). ${ }^{19}$ The conventional setup comprises five vessels, simulating the stomach, small intestine and three colon regions. In this experiment, only the ascending colon was simulated as this is the most relevant region regarding colonisation of AIEC which is known to predominantly colonise the distal ileum and proximal colon (Figure 3). The mucosal environment in the colon compartments $(500 \mathrm{ml})$ consisted of 80 mucin agar-covered microcosms (AnoxKaldnes K1 carrier, AnoxKaldnes AB, Lund, Sweden). Every $48 \mathrm{~h}, 50 \%$ of the mucin 
agar-covered microcosms has been replaced by fresh microcosms. The lid of each reactor is slightly opened under constant nitrogen flush and a net containing 40 of the 80 microcosms is quickly replaced by a new one. Old microcosms are then stored for further analysis. The colon regions were simultaneously inoculated with $40 \mathrm{ml}$ of faecal slurry derived from fresh stools of a healthy human volunteer (26 years). Faecal samples were 1:5 $(\mathrm{w} / \mathrm{v})$ diluted in phosphate buffer $(0.05 \mathrm{M}, \mathrm{pH}=7)$ containing $1 \mathrm{~g} / \mathrm{l}$ sodium thioglycolate, followed by homogenisation with a Stomacher Lab-Blender (Seward Medical, London, UK) and centrifugation to remove particulate material (500 g, $1 \mathrm{~min}$ ). By imposing identical conditions, similar microbial composition and activity was achieved in the five parallel ascending colon vessels. $^{36}$ Three times per day, $140 \mathrm{ml}$ nutritional medium and $60 \mathrm{ml}$ pancreatic juice were added to the stomach and small intestine, respectively.

\section{Experimental design M-SHIME study}

Based on initial screenings, three treatments were applied in a third experiment, a long-term M-SHIME study with five colon vessels, organised as follows (Figure 3 ). The first unit consisted of a conventional setup that only simulates luminal microbes (=L-SHIME), while the second also simulates mucosal microbes (=M-SHIME). These two units allowed evaluating the importance of a mucosal environment for the colonisation of AIEC. The M-SHIME unit also served as a control to the other three units that also contained a mucosal environment $(=\mathrm{M}$-SHIME) but that were treated with L. reuteri 1063, LC-AX and IN. After inoculation, human faecal microbes were stabilised during 14 days, after which the treatment started. For LC-AX and IN, $3 \mathrm{~g}$ per day was added, while for L. reuteri $106310 \mathrm{ml}$ of an overnight-grown culture, washed in PBS, was applied. On day 22, 23, 24 and $25,10 \mathrm{ml}$ of an overnight-grown culture of AIEC, washed in PBS, was inoculated. The experiment was stopped at day 31. On the days that the mucosal environment was renewed (day 22, 24, 27, 29 and 31), AIEC was enumerated in the luminal and mucosal microbiota by means of plating.

\section{Microbial community analysis: plate counts, flow cytometry and DGGE}

AIEC numbers were determined by plating on a specific agar, i.e., MacConkey (Oxoid) supplemented with $50 \mathrm{mg} / \mathrm{l}$ ampicillin and $20 \mathrm{mg} / \mathrm{l}$ erythromycin. Samples were serially diluted in saline solution $(8.5 \mathrm{~g} / \mathrm{l} \mathrm{NaCl})$, after which plates were inoculated and incubated aerobically at $37^{\circ} \mathrm{C}$. Pure cultures of AIEC and probiotic bacteria were quantified using flow cytometry after live-dead staining as described earlier. ${ }^{19}$

DNA extraction according to Boon et al. ${ }^{37}$ was performed on the pellet of $1 \mathrm{ml}$ suspension for luminal samples or $0.5 \mathrm{~g}$ mucin agar for mucosal samples. Denaturing Gradient Gel Electrophoresis (DGGE) was applied to separate PCR products of the 16S rRNA genes of Bifidobacterium sp. and the total bacterial community. For the total community, general bacterial primers $338 \mathrm{~F}-\mathrm{GC}$ and $518 \mathrm{R}$ were used ${ }^{38}$ while the bifidobacteria required a first PCR using specific primers ${ }^{39}$ and a second PCR on the 1:100 diluted PCR product using the general bacterial primers. Polyacrylamide gels (8\%) had a denaturing gradient ranging from 45 to $60 \%$ for the total microbiota and from 50 to $65 \%$ for the bifidobacteria. Gels were run using an Ingeny PhorU apparatus (Ingeny International, Goes, the Netherlands). Normalisation and further analysis was carried out using the BioNumerics software version 5.10 (Applied Maths, Sint-Martens-Latem, Belgium). Specific bands were cut and sequenced by ITT Biotech-Bioservice (Bielefeld, Germany). Sequence data have been submitted to the EMBL database (accession numbers HE985181-HE985183).

Metabolic activity analysis: lactic acid, SCFA, 3-HPA, $\mathrm{NH}_{4}^{+}, 3-\mathrm{HPA}$ and 1,3-PDO

Lactic acid was measured using a D/L-lactic acid kit (R-Biopharm, Mannheim, Germany), according to the manufacturer's protocols. pH was measured and used to calculate undissociated lactic acid as this is the antimicrobial lactic acid fraction: undissociated lactic acid $(\%)=1 /[1+10 \wedge$ $(\mathrm{pH}-\mathrm{pKa})] \times 100 \%$. Further, $3-\mathrm{HPA}$ and $1,3-\mathrm{PDO}$ were quantified by means of HPLC to indicate the reuterin production, and SCFA by means of GC as reported by De Weirdt et al. ${ }^{40}$ Using a Kjeltec Auto Distillation (FOSS Benelux, Amersfoort, the Netherlands), $\mathrm{NH}_{4}^{+}$in the sample was liberated as $\mathrm{NH}_{3}$ by the addition of $\mathrm{MgO}$. Released $\mathrm{NH}_{3}$ was distilled from the sample into a boric acid solution, which was back-titrated using a 665 Dosimat (Metrohm, Berchem, Belgium) and 686 Titroprocessor (Metrohm).
Statistics

All data were analysed using the SPSS 16 software (SPSS, Chicago, IL, USA). Before investigating probability of intergroup differences, normality was studied with a Kolmogorov-Smirnov test. Normal distributed data were further analysed in a one-way ANOVA test, followed by post hoc Bonferroni (equal variances) or Dunnett's T3 (non-equal variances) analysis. Non-normal distributed data were tested for differences using a Kruskal-Wallis with Mann-Whitney test. Differences were considered significant when $P<0.05$.

\section{ACKNOWLEDGEMENTS}

M.M. and P.V.d.A. are Postdoctoral Fellows, supported by FWO-Vlaanderen (Research Foundation of Flanders, Belgium). R.D.W. is a postdoctoral fellow supported by a Concerted Research Action of the Flemish Community (GOA) (BOF12/GOA/008). Finally, this work was partially supported by an SBO project (100016) from the Agency for Innovation by Science and Technology (IWT).

\section{CONTRIBUTIONS}

P.V.d.A., M.D., R.D.W. and V.J. contributed to the experimental part, while P.V.d.A. M.M., S.P. and T.V.d.W. designed the experiments and wrote this manuscript.

\section{COMPETING INTERESTS}

The authors declare no conflict of interest.

\section{REFERENCES}

1. Zoetendal, E. G. et al. Mucosa-associated bacteria in the human gastrointestinal tract are uniformly distributed along the colon and differ from the community recovered from feces. Appl. Environ. Microbiol. 68, 3401-3407 (2002).

2. Van den Abbeele, P., Van de Wiele, T., Verstraete, W. \& Possemiers, S. The host selects mucosal and luminal associations of co-evolved gut microbes: a novel concept. FEMS Microbiol. Rev. 35, 681-704 (2011).

3. Meyer-Hoffert, U. et al. Secreted enteric antimicrobial activity localises to the mucus surface layer. Gut 57, 764-771 (2008).

4. Roos, S. \& Jonsson, H. A high-molecular-mass cell-surface protein from Lactobacillus reuteri 1063 adheres to mucus components. Microbiology 148, 433-442 (2002).

5. Koropatkin, N. M., Cameron, E. A. \& Martens, E. C. How glycan metabolism shapes the human gut microbiota. Nat. Rev. Microbiol. 10, 323-335 (2012).

6. Collado, M. C. et al. Antimicrobial peptides are among the antagonistic metabolites produced by Bifidobacterium against Helicobacter pylori. Int. J. Antimicrob. Agents 25, 385-391 (2005).

7. Salminen, S. et al. Functional food science and gastrointestinal physiology and function. Br. J. Nutr. 80, S147-S171 (1998).

8. Gibson, G. R. \& Roberfroid, M. B. Dietary modulation of the human colonic microbiota-introducing the concept of prebiotics. J. Nutr. 125, 1401-1412 (1995).

9. Hooper, L. V., Xu, J., Falk, P. G., Midtvedt, T. \& Gordon, J. I. A molecular sensor that allows a gut commensal to control its nutrient foundation in a competitive ecosystem. Proc. Natl Acad. Sci. USA 96, 9833-9838 (1999).

10. Bernet, M. F., Brassart, D., Neeser, J. R. \& Servin, A. L. Lactobacillus-acidophilus LA-1 binds to cultured human intestinal-cell lines and inhibits cell attachment and cell invasion by enterovirulent bacteria. Gut 35, 483-489 (1994).

11. Willing, B. et al. Twin studies reveal specific imbalances in the mucosa-associated microbiota of patients with ileal Crohn's disease. Inflamm. Bowel Dis. 15, 653-660 (2009).

12. Willing, B. P. et al. A Pyrosequencing study in twins shows that gastrointestinal microbial profiles vary with inflammatory bowel disease phenotypes. Gastroenterology 139, 1844-1854.e1841 (2010).

13. Darfeuille-Michaud, A. et al. High prevalence of adherent-invasive Escherichia coli associated with ileal mucosa in Crohn's disease. Gastroenterology 127, 412-421 (2004).

14. Gibold, L. et al. The Vat-AIEC protease promotes crossing of the intestinal mucus layer by Crohn's disease-associated Escherichia coli. Cell. Microbiol. 18, 617-631 (2016).

15. Ouwehand, A. C., Kirjavainen, P. V., Gronlund, M. M., Isolauri, E. \& Salminen, S. J. Adhesion of probiotic micro-organisms to intestinal mucus. Int. Dairy J. 9, 623-630 (1999). 
16. Van den Abbeele, P. et al. In vitro model to study the modulation of the mucin-adhered bacterial community. Appl. Microbiol. Biotechnol. 83, 349-359 (2009).

17. Tsilingiri, K. et al. Probiotic and postbiotic activity in health and disease: comparison on a novel polarised ex-vivo organ culture model. Gut 61, 1007-1015 (2012).

18. Laparra, J. M. \& Sanz, Y. Comparison of in vitro models to study bacterial adhesion to the intestinal epithelium. Lett. Appl. Microbiol. 49, 695-701 (2009).

19. Van den Abbeele, P. et al. Incorporation of a mucosal environment in a dynamic gut model results in a more representative colonization by lactobacilli. Microb. Biotechnol. 5, 106-115 (2011).

20. Van den Abbeele, P. et al. Butyrate-producing Clostridium cluster XIVa species specifically colonize mucins in an in vitro gut model. ISME J. 7, 949-961 (2013).

21. Darfeuille-Michaud, A. et al. Presence of adherent Escherichia coli strains in ileal mucosa of patients with Crohn's disease. Gastroenterology 115, 1405-1413 (1998).

22. Van den Abbeele, P. et al. Arabinoxylans and inulin differentially modulate the mucosal and luminal gut microbiota and mucin-degradation in humanized rats. Environ. Microbiol. 13, 2667-2680 (2011).

23. Kankainen, M. et al. Comparative genomic analysis of Lactobacillus rhamnosus GG reveals pili containing a human- mucus binding protein. Proc. Natl Acad. Sci. USA 106, 17193-17198 (2009).

24. Barnich, N., Boudeau, J., Claret, L. \& Darfeuille-Michaud, A. Regulatory and functional co-operation of flagella and type 1 pili in adhesive and invasive abilities of AIEC strain LF82 isolated from a patient with Crohn's disease. Mol. Microbiol. 48, 781-794 (2003).

25. Martinez-Medina, M. et al. Molecular diversity of Escherichia coli in the human gut: New ecological evidence supporting the role of adherent-invasive E. coli (AIEC) in Crohn's disease. Inflamm. Bowel Dis. 15, 872-882 (2009).

26. Lee, H. Y. et al. Prophylactic uses of probiotics as a potential alternative to antimicrobials in food animals. Food Sci. Biotechnol. 17, 191-194 (2008).

27. Khedkar, J. N., Dave, J. M. \& Sannabhadti, S. S. Antibacterial activity associated with Bifidobacterium adolescentis. J. Food Sci. Technol. 35, 527-529 (1998).

28. Stöber, H., Maier, E. \& Schmidt, H. Protective effects of Lactobacilli, Bifidobacteria and Staphylococci on the infection of cultured HT29 cells with different enterohemorrhagic Escherichia coli serotypes are strain-specific. Int. J. Food Microbiol. 144, 133-140 (2010).

29. Axelsson, L. T., Chung, T. C., Dobrogosz, W. J. \& Lindgren, S. E. Production of a broad spectrum antimicrobial substance by Lactobacillus reuteri. Microb. Ecol. Health Dis. 2, 131-136 (1989).

30. Talarico, T. L., Casas, I. A., Chung, T. C. \& Dobrogosz, W. J. Production and isolation of reuterin, a growth inhibitor produced by Lactobacillus reuteri. Antimicrob. Agents Chemother. 32, 1854-1858 (1988).
31. Cleusix, V., Lacroix, C., Vollenweider, S., Duboux, M. \& Le Blay, G. Inhibitory activity spectrum of reuterin produced by Lactobacillus reuteri against intestinal bacteria. BMC Microbiol. 7, 101 (2007).

32. Cleusix, V., Lacroix, C., Vollenweider, S. \& Le Blay, G. Glycerol induces reuterin production and decreases Escherichia coli population in an in vitro model of colonic fermentation with immobilized human feces. FEMS Microbiol. Ecol. 63, 56-64 (2008).

33. Marchesi, J. R. et al. Rapid and noninvasive metabonomic characterization of inflammatory bowel disease. J. Proteome Res. 6, 546-551 (2007).

34. Gibson, G. R., McCartney, A. L. \& Rastall, R. A. Prebiotics and resistance to gastrointestinal infections. Br. J. Nutr. 93 Suppl 1, S31-S34 (2005).

35. Vollenweider, S. \& Lacroix, C. 3-Hydroxypropionaldehyde: applications and perspectives of biotechnological production. Appl. Microbiol. Biotechnol. 64, 16-27 (2004).

36. Van den Abbeele, P. et al. Microbial community development in a dynamic gut model is reproducible, colon-region specific and selects for Bacteroidetes and Clostridium cluster IX. Appl. Environ. Microbiol. 76, 5237-5246 (2010).

37. Boon, N., Top, E. M., Verstraete, W. \& Siciliano, S. D. Bioaugmentation as a tool to protect the structure and function of an activated-sludge microbial community against a 3-chloroaniline shock load. Appl. Environ. Microbiol. 69, 1511-1520 (2003).

38. Muyzer, G., Dewaal, E. C. \& Uitterlinden, A. G. Profiling of complex microbialpopulations by denaturing gradient gel-electrophoresis of polymerase chain reaction-amplified genes-coding for $16 \mathrm{~S}$ ribosomal-RNA. Appl. Environ. Microbiol. 59, 695-700 (1993).

39. Satokari, R. M., Vaughan, E. E., Akkermans, A. D. L., Saarela, M. \& de Vos, W. M. Bifidobacterial Diversity in Human Feces Detected by Genus-Specific PCR and Denaturing Gradient Gel Electrophoresis. Appl. Environ. Microbiol. 67, 504-513 (2001).

40. De Weirdt, R. et al. Human faecal microbiota display variable patterns of glycerol metabolism. FEMS Microbiol. Ecol. 74, 601-611 (2010).

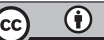

This work is licensed under a Creative Commons Attribution 4.0 International License. The images or other third party material in this article are included in the article's Creative Commons license, unless indicated otherwise in the credit line; if the material is not included under the Creative Commons license, users will need to obtain permission from the license holder to reproduce the material. To view a copy of this license, visit http://creativecommons.org/licenses/ by/4.0/

(c) The Author(s) 2016

Supplementary Information accompanies the paper on the npj Biofilms and Microbiomes website (http://www.nature.com/npjbiofilms) 